\title{
Experimental Demonstration of a Machine Learning-Based in-band OSNR Estimator from Optical Spectra
}

\author{
Fabiano Locatelli ${ }^{1,2 *}$, Konstantinos Christodoulopoulos ${ }^{2}$, Josep M. Fàbrega ${ }^{1}$, Michela Svaluto \\ Moreolo $^{1}$, Laia Nadal ${ }^{1}$, Salvatore Spadaro ${ }^{3}$ \\ ${ }^{1}$ Centre Tecnologic de Telecomunicacions de Catalunya (CTTC/CERCA), Castelldefels (Barcelona), Spain; ${ }^{2}$ Nokia Bell \\ Labs, Stuttgart, Germany; ${ }^{3}$ Universitat Politècnica de Catalunya (UPC), Barcelona, Spain \\ *fabiano.locatelli@cttc.es
}

\begin{abstract}
Channel spectral monitors are becoming a cost effective solution to improve the management, resiliency and efficiency of next generation optical transport networks. We experimentally demonstrate a technique based on machine learning (ML) for the in-band estimation of amplified spontaneous emission (ASE) noise and filter 3-dB bandwidth, using optical spectra acquired after the reconfigurable optical add/drop multiplexers (ROADMs) filters. We assess the performance of the proposed method, considering laser drift and filters bandwidth tightening scenarios, showing quite good estimation accuracy under such conditions.
\end{abstract}

Keywords-Machine learning, optical performance monitoring, optical spectrum

\section{INTRODUCTION}

Optical performance monitoring (OPM) has a key role in guaranteeing the end-to-end quality of transmission (QoT) and quality of service (QoS) of the network. Among all the parameters that can be monitored by OPM, optical signal to noise ratio (OSNR) is one of the most important, since it is transparent to both the bit rate and the modulation format and it is directly related to the bit error rate (BER) [1]. Monitoring OSNR is key for optimizing channels when planning or upgrading the network. For non-operating channels, this is typically done by turning $\mathrm{ON}$ and OFF the connection [2]. However, such monitoring approach is not feasible in operating channels. Monitoring OSNR degradations that may occur in an operating network in a distributed/per link or node fashion, as shown in Fig. 1, can feed a closed control loop. Combined with appropriate algorithms it can allow the identification of link-degrading impairments, helping to prevent soft-failures or to dynamically optimize the network [3]. To do so, cheap and small form factor OSNR monitoring solutions must be developed. Furthermore, while passing through the optical nodes, the reconfigurable optical add/drop multiplexers (ROADMs), the connection will cross a certain number of optical filters. Conventional OSNR monitoring techniques struggle in presence of strong filtering effects, which make the noise level identification difficult [4]. Thus, the OSNR must be measured in-band [5].

In an optical network, an ideal scenario would include omnipresent and powerful OPMs, i.e. before and after every network element. However, in reality this solution is not feasible because of its cost. We envision the use of cheap/low

This work was partially funded by the ONFIRE project supported by EU Horizon 2020 research and innovation programme under the Marie Skłodowska-Curie grant agreement No. 765275. resolution optical spectrum analyzers (OSA) as OPM to be used for OSNR monitoring and other applications [6]. It stands to reason that the most appropriate position of the OSA would be close to the ROADMs, at the ingress ports (before the ingress wavelength selective switches -WSSs-), in the WSS interconnections, and at the egress ports (after the egress WSS). Fig. 2 shows these possible locations of the OSAs. Again due to cost reasons, we might have to limit/select their placement. Moreover, in a disaggregated network scenario, the OSAs placement could be non-uniform, i.e. varying from node to node [7], [8]. Indeed some nodes could be equipped with monitoring only at their input, some others at their output and some could not be monitored at all.

In [9], the authors identified support vector machine (SVM) regression as the most promising machine learning (ML) approach for OSNR estimation. However, [9] assumed a wide-band signal and in the majority of their evaluations used spectra coming from simulations. In our previous work [10], we also examined the performance of ML based models for in-band OSNR estimation, in particular SVM regression and a Gaussian process regression (GPR) model. However in [10], our experimental setup didn't include any optical filter; we post-processed the set of collected optical spectra to replicate it. Moreover, [10] was lacking the verification of our proposed method under strong filtering conditions.

In this paper, we further assess the GPR ML-based in-band OSNR estimation method proposed in [10] with a new set of experimental collected spectral data. We have chosen GPR over SVM model, because of the results obtained in our previous work [10]. The new experimental setup included an optical filter, which we configured appropriately by reducing and shifting its bandwidth to replicate two common impairments: the laser drift and the filter tightening effects. In our experiments, we placed the OSA after the optical filter, reproducing the scenario in which the optical monitors are present only after the nodes. Therefore, the monitored spectra suffer from strong filtering making it hard to identify the actual noise levels, as previously mentioned. The proposed solution showed quite good estimation accuracy under such realistic conditions. In addition, we demonstrate a 3-dB filter bandwidth estimator based on the GPR model.

\section{EXPERIMENTAL ACQUISITION OF THE OPTICAL SPECTRA AND THEIR PROCESSING}

Fig. 3 depicts the experimental setup we used to capture the spectral data and replicate scenarios were the monitored signal suffers from strong filtering effects, i.e. the OSAs are 


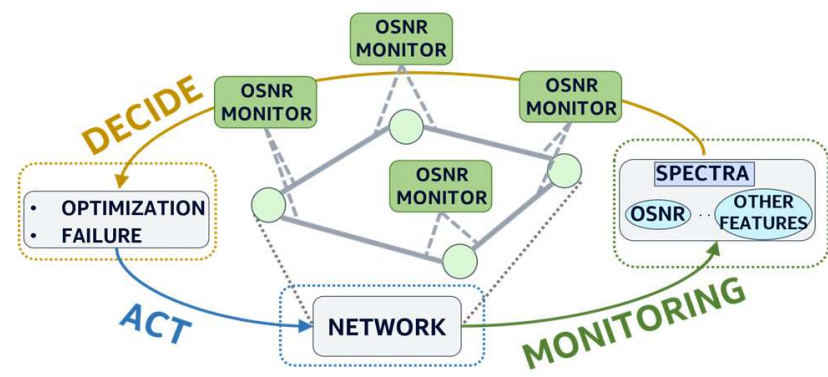

Fig. 1. Network monitoring/decide/act loop.

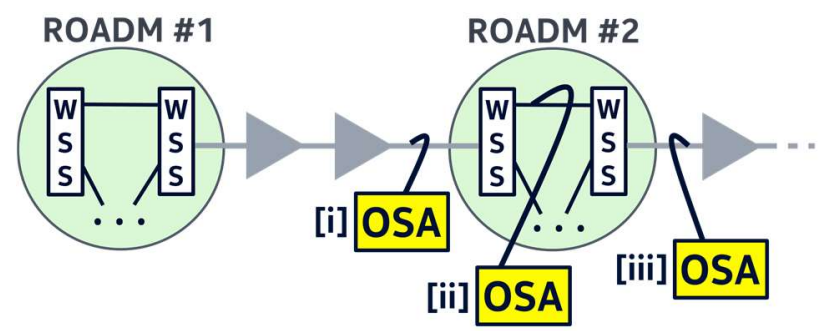

Fig. 2. OSAs placement: [i] at the ingress of the ROADM; [ii] in the WSSs interconnection; [iii] at the egress of the ROADM.

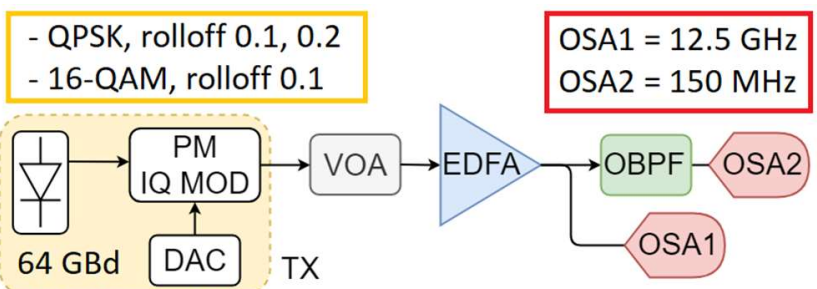

Fig. 3. Experimental setup. PM-IQ-MOD: polarization multiplexed-IQ-modulator, DAC: digital-to-analog converter, TX: transmitter, VOA: variable optical attenuator, EDFA: erbiumdoped fiber amplifier, OBPF: optical band-pass filter, OSA: optical spectrum analyzer.

placed inside or after the ROADMs (positions ii and iii in Fig. 2 ). By means of a tunable laser working at $1550.116 \mathrm{~nm}$, we generated a $64 \mathrm{GBd}$ polarization multiplexed-quadrature phase shift keying (PM-QPSK) modulated signal, with 0.1 roll-off factor. We did all the measurements in a back to back (B2B) scenario. After the transmitter, we placed a variable optical attenuator (VOA) and an erbium-doped fiber amplifier (EDFA) operating in automatic power control mode. With this configuration, we obtained 9 different OSNR levels, which ranged between 13 and $28 \mathrm{~dB}$. Right after the EDFA we placed an OSA with resolution bandwidth of $12.5 \mathrm{GHz}$, which gave us the amplified spontaneous emission (ASE) noise level of the signals to be used as reference values. Then, the signal passes through an optical band-pass filter (OBPF) with 75 $\mathrm{GHz}$ pass-band, centered at the laser's wavelength. Note that for the used $64 \mathrm{GBd}$ signal, the control plane would configure the filter bandwidth at $75 \mathrm{GHz}$. Finally, by means of a second OSA with spectral resolution of $150 \mathrm{MHz}$, we acquired the spectra of the optically filtered signal. By shifting the central wavelength of the OBPF and tuning its bandwidth, we reproduced two typical optical impairments: laser drift and filter tightening. In particular, we considered 5 filter's bandwidths (i.e. 75, 72, 69, 66, $63 \mathrm{GHz}$ ) and for each one of them 4 shift scenarios: $\pm 1 \mathrm{GHz}$ and $\pm 2 \mathrm{GHz}$. With this configuration, we acquired 1125 optical spectra. We repeated each acquisition 5 times and averaged them during the postprocessing phase. In addition, we replicated the same scenario with the same modulation format (i.e. PM-QPSK) and a rolloff factor of 0.2. In this situation, we acquired 180 spectra with the filter bandwidth set at 75, 75+2, 69 and 69-2 GHz. Finally, we used the same setup with a PM-16 quadrature amplitude modulation (QAM) format and 0.1 roll-off factor, and again captured 180 optical spectra with the filter bandwidth set at $75,75+2,69$ and 69-2 GHz. Once we collected the spectra, to replicate the situation of a wavelength division multiplexing (WDM) network, we normalized their peak to $0 \mathrm{dBm}$. The results of this processing are shown in Fig. 4a and Fig. 4b, where the optical spectra of the 9 VOA levels are depicted before and after the filtering process, respectively, for $75 \mathrm{GHz}$ OBPF bandwidth.

Following a similar notation to [10], we represent each acquired optical spectrum with the vector $\boldsymbol{s}$ of length $n$. When collected, the original length of the spectral data was equal to 50000 ( $75 \mathrm{GHz}$ channel at $150 \mathrm{MHz}$ granularity). We then applied a cut, which just maintained the rising and the falling part of each spectrum and resulted in a reduction of their length $n$ to 800 samples. Fig. 4c shows the filtered spectra after the cutting process at different filter bandwidths. We observe a slight asymmetry in the spectra, which originates from the imperfect alignment between the laser and the OBPF central frequency, although both were set to the same values in the lab. Such issues are quite typical in real networks and exacerbate in long paths with several filters in cascade, which motivated our study of further misalignment levels. Finally, we associated each spectrum $\boldsymbol{s}$ to its reference ASE noise value $y$, measured with OSA1, and to its reference 3 -dB filter bandwidth value $z$

\section{OSNR AND 3-DB FILTER BANDWIDTH ESTIMATOR}

GPR model is a non-parametric kernel-based ML technique, which we trained using the squared exponential kernel function. We use the GPR model to first find a mapping $g$ between the connection spectrum $\mathbf{s}$ and its 3 -dB filter bandwidth $z$, and then a further mapping $f$ between the same connection spectrum $\boldsymbol{s}$ and its noise value $y$, that are $z=g(\boldsymbol{s})$ and $y=f(\boldsymbol{s})$, respectively. We first define a matrix $\boldsymbol{S}_{\boldsymbol{d}}$, with dimensions $n \times m_{1}$, to represent the $m_{1}$ acquired optical spectra $\mathbf{s}$ of length $n$ with the same transmission parameters $d=(q, a)$, where $q$ represents the baud rate and $a$ the roll-off factor. Then, we define the matrix $\boldsymbol{S}_{\boldsymbol{c}}$, with dimensions $n \times m_{2}$, and parameters $c=(l, q, a, b)$, where $l$ represents the modulation format and $b$ the $3-\mathrm{dB}$ filter bandwidth of each spectrum. Finally, with the two vectors $\boldsymbol{z}_{\boldsymbol{d}}$ and $\boldsymbol{y}_{c}$, of length $m_{1}$ and $m_{2}$, we denote the reference $3-\mathrm{dB}$ filter bandwidth and the reference noise values of each spectrum, respectively. To approximate the two estimation functions $g$ and $f$, we implemented two ML models $G_{d}$ and $F_{c}$, specific for channels with parameters $d$ and $c$. We trained $G_{d}$ with the input set $\left(\boldsymbol{S}_{\boldsymbol{d}}, \boldsymbol{z}_{\boldsymbol{d}}\right)$ and $F_{c}$ with the input set $\left(\boldsymbol{S}_{c}, \boldsymbol{y}_{c}\right) . d$ and $c$ have different parameters because $G_{d}$ generalize differently from $F_{c}$. Having no hyperparameter to tune, we didn't use any validation set. The training phase has the purpose of identifying the two ML models that minimize some functions of the estimation error, such as the mean squared error (MSE) function. In fact, if we define the estimated 3-dB filter bandwidth as $\widehat{\boldsymbol{z}}_{\boldsymbol{d}}=G_{d}\left(\boldsymbol{S}_{\boldsymbol{d}}\right)$ and the estimated ASE noise values as $\widehat{\boldsymbol{y}}_{\boldsymbol{c}}=F_{c}\left(\boldsymbol{S}_{\boldsymbol{c}}\right)$, we can also define their respective estimation errors as $\boldsymbol{\varepsilon}_{\boldsymbol{d}}^{\boldsymbol{z}}=\widehat{\mathbf{z}}_{\boldsymbol{d}}-\boldsymbol{z}_{\boldsymbol{d}}$ and $\boldsymbol{\varepsilon}_{\boldsymbol{c}}^{\boldsymbol{y}}=\hat{\boldsymbol{y}}_{\boldsymbol{c}}-\boldsymbol{y}_{\boldsymbol{c}}$. Once the ML models are trained with the spectra $\boldsymbol{S}_{\boldsymbol{d}}$ and $\boldsymbol{S}_{\boldsymbol{c}}$ and their reference values $\boldsymbol{z}_{\boldsymbol{d}}$ and $\boldsymbol{y}_{\boldsymbol{c}}$, they will be able to estimate from the spectrum $\boldsymbol{s}$ of an operating 

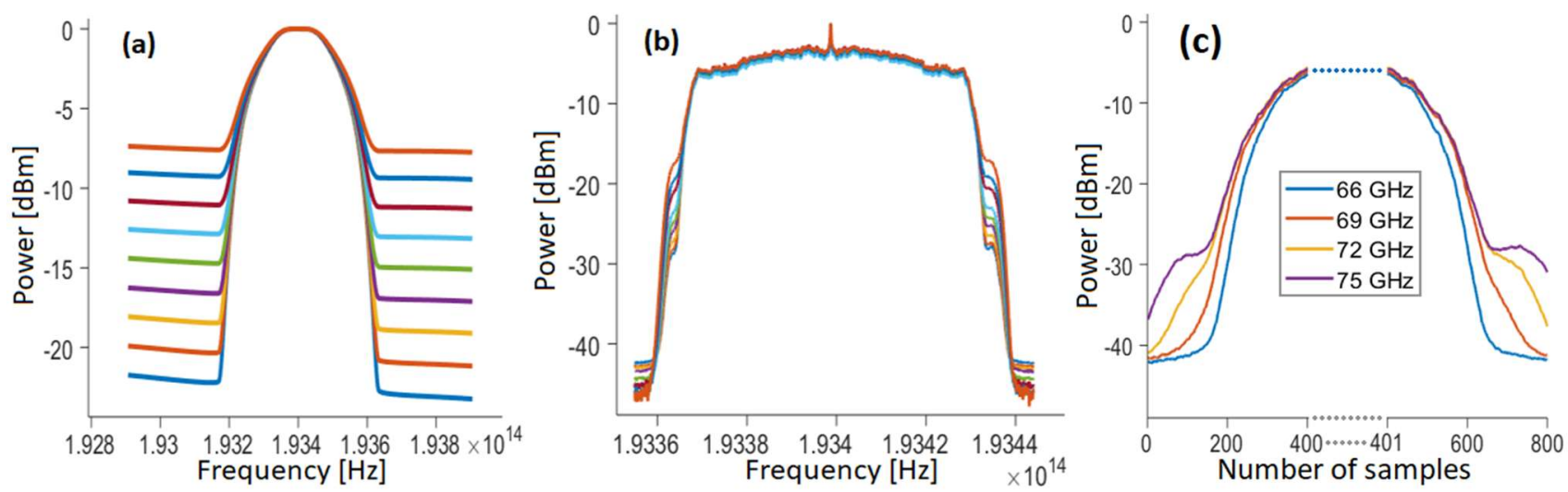

Fig. 4. (a) 9 VOA levels optical spectra collected by OSA1, before the optical filtering phase; (b) Normalized filtered spectra with filter 3-dB bandwidth at $75 \mathrm{GHz}$ and 9 different VOA levels; (c) Spectra filtered with 75, 72, 69 and $66 \mathrm{GHz}$ 3-dB filter bandwidth, after the cutting process.
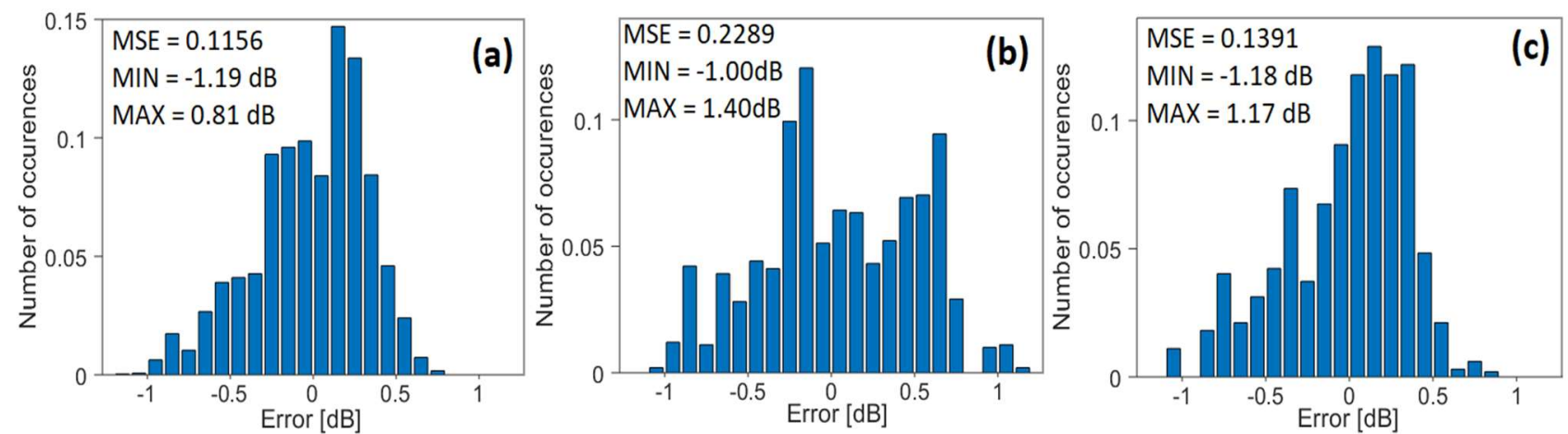

Fig. 5. Probability density functions (PDFs) of the noise estimation error for the $69 \mathrm{GHz}$ filter bandwidth scenarios: (a) $l=$ QPSK, $a=$ 0.1 ; (b) $l=$ QPSK, $a=0.2$; (c) $l=16-\mathrm{QAM}, a=0.1$.

TABLE I

SUMMARY OF RESULTS FOR THE ASE NOISE ESTIMATION

\begin{tabular}{|c|c|c|c|c|c|c|}
\hline $\begin{array}{c}\text { Mod. } \\
\text { format (l) }\end{array}$ & $\begin{array}{c}\text { Symbol } \\
\text { rate } \\
\text { (q) }\end{array}$ & $\begin{array}{l}\text { Roll-off } \\
\text { factor } \\
\text { (a) }\end{array}$ & $\begin{array}{c}3 d B \\
\text { filter } \\
\text { bandwi } \\
d t h(b)\end{array}$ & $M S E$ & $\begin{array}{l}M I N \\
{[d B]}\end{array}$ & $\begin{array}{l}M A X \\
{[d B]}\end{array}$ \\
\hline \multirow{5}{*}{$\begin{array}{c}\text { PM- } \\
\text { QPSK }\end{array}$} & \multirow{5}{*}{$\begin{array}{c}64 \\
\text { GBd }\end{array}$} & \multirow{5}{*}{0.1} & $63 \mathrm{GHz}$ & 0.427 & -1.880 & 1.693 \\
\hline & & & $66 \mathrm{GHz}$ & 0.187 & -1.573 & 1.430 \\
\hline & & & $69 \mathrm{GHz}$ & 0.115 & -1.194 & 0.813 \\
\hline & & & $72 \mathrm{GHz}$ & 0.055 & -0.966 & 0.549 \\
\hline & & & $75 \mathrm{GHz}$ & 0.029 & -0.627 & 0.497 \\
\hline \multirow{2}{*}{$\begin{array}{c}\text { PM- } \\
\text { QPSK }\end{array}$} & \multirow{2}{*}{$\begin{array}{c}64 \\
\text { GBd } \\
\end{array}$} & \multirow{2}{*}{0.2} & $69 \mathrm{GHz}$ & 0.228 & -1.167 & 1.207 \\
\hline & & & $75 \mathrm{GHz}$ & 0.172 & -0.992 & 0.868 \\
\hline \multirow{2}{*}{$\begin{array}{c}\text { PM- } \\
\text { 16QAM }\end{array}$} & \multirow{2}{*}{$\begin{array}{c}64 \\
\text { GBd }\end{array}$} & \multirow{2}{*}{0.1} & $69 \mathrm{GHz}$ & 0.139 & -1.280 & 1.172 \\
\hline & & & $75 \mathrm{GHz}$ & 0.030 & -0.923 & 0.895 \\
\hline
\end{tabular}

channel with the same set of parameters $d$ or $c$, its 3-dB filter bandwidth $\widehat{\boldsymbol{z}}_{\boldsymbol{d}}$ and its ASE noise value $\widehat{\boldsymbol{y}}_{\boldsymbol{c}}$.

\section{RESUlT AND DisCUSSION}

For both the models $G_{d}$ and $F_{c}$, we used the $\sim 85 \%$ of the total data to train the algorithm and the remaining $\sim 15 \%$ to test it. Furthermore, to evaluate their estimation accuracy, we randomly shuffled the training and the testing sets 200 times, trained a different ML model each time and tested it with the different corresponding test sets. We first evaluated the 3-dB filter bandwidth estimator $G_{d}$, bearing in mind that such estimation could be performed also with conventional algorithms. In all the cases, the maximum estimation errors $\boldsymbol{\varepsilon}_{\boldsymbol{d}}^{\boldsymbol{z}}$ were below $1 \mathrm{GHz}$. Then, we evaluated the performance of the ASE noise estimation model $F_{c}$. The MSE, the minimum (MIN) and the maximum (MAX) errors related to each set of parameters $c$ are summarized in Table I. We observed a maximum estimation error $\varepsilon_{c}^{y}$ lower than $1.7 \mathrm{~dB}$ in all the scenarios. In particular, for wider filter bandwidth the estimation is more precise because the noise level is not cut by the filter. In such situations, traditional method can also be adopted. On the other hand, when it comes to narrower 3-dB bandwidth, ML techniques can still understand the filter effects on the noise, while traditional methods struggle. Fig. 5 shows the probability density function (PDF) of the noise estimation error, for the $b=69 \mathrm{GHz}$ cases of each modulation format/roll-off factor scenario. We also trained and tested the $F_{c}$ model without considering the bandwidth parameter $b$ in the set $c$. The obtained MAX error was $\sim 2.5 \mathrm{~dB}$. This performance degradation was caused by the lack of a priori knowledge of the filter 3-dB bandwidth, a key parameter for ASE noise estimation.

\section{CONCLUSION}

We presented a method based on GPR model to estimate the filtered 3-dB bandwidth and the ASE noise values from optical spectra that suffer from strong filtering effects, e.g. from an OSA placed after the filter. On experimental collected spectra our method achieved a good estimation accuracy in all examined scenarios; the 3-dB filter bandwidth estimation maximum error was $1 \mathrm{GHz}$, while the ASE noise estimator achieved a maximum error lower than $1.2 \mathrm{~dB}$ for filter bandwidths larger or equal to $69 \mathrm{GHz}$ for a $64 \mathrm{GBd}$ signal.

\section{ACKNOWLEDGMENT}

The authors would like to thank Fred Buchali, Karsten Schuh, Roman Dischler and Ankush Mahajan for lab support. 


\section{REFERENCES}

[1] C. C. K. Chan, Optical Performance Monitoring, Elsevier, 2010.

[2] ITU G.697, "Optical monitoring for dense wavelength division multiplexing systems", 2016

[3] K. Christodoulopoulos et al., "Toward Efficient, Relieable, and Autonomous Optical Networks: the ORCHESTRA Solution [Invited]," IEEE/OSA JOCN, vol. 11, no. 9, Sep. 2019.

[4] J. M. Fàbrega et al., "On the filter narrowing issues in elastic optical networks," IEEE/OSA JOCN, vol. 8, no. 7, Jul. 2016.

[5] IEC TR 61282-12, "Fibre Optic Communication System Design Guides - Part 12: In-Band Optical Signal-To-Noise Ratio (OSNR)", 2016 .
[6] L. Velasco, B. Shariati, A. P. Vela, J. Comellas, and M. Ruiz, "Learning from the Optical Spectrum: Soft-Failure Identification and Localization [Invited]," in OFC 2018.

[7] Open and Disaggregated Transport Network (ODTN) Project [On-line] www.opennetworking.org/odtn/.

[8] E. Riccardi et al., "An Operator view of the Introduction of White Boxes into Optical Networks," IEEE/OSA JLT, vol. 36, no. 15, Aug. 2018.

[9] D. Wang et al., "Machine Learning-Based Multifunctional Optical Spectrum Analysis Technique," IEEE Access, vol. 7, Jan. 2019.

[10] F. Locatelli et al., "Machine Learning-Based in-band OSNR Estimation from Optical Spectra," IEEE PTL, vol. 31, no. 24, Dec. 2019. 